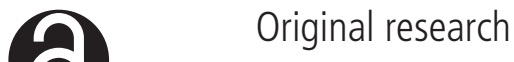

\section{Absence of pontine perforators in vertebrobasilar dolichoectasia on ultra-high resolution cone-beam computed tomography}

\author{
Tomas Dobrocky 다, ' Eike I Piechowiak 다, ' Johannes Goldberg, ${ }^{2}$ \\ Enrique Barvulsky Aleman, ${ }^{1}$ Patrick Nicholson, ${ }^{3}$ Jeremy Lynch, ${ }^{3}$ David Bervini, ${ }^{2}$ \\ Johannes Kaesmacher (10 , ${ }^{1}$ Ronit Agid, ${ }^{3}$ Timo Krings, ${ }^{3}$ Andreas Raabe, ${ }^{2}$ Jan Gralla, ${ }^{1}$ \\ Vitor M Pereira, ${ }^{3}$ Pasquale Mordasini ${ }^{1}$
}

${ }^{1}$ Department of Diagnostic and Interventional Neuroradiology, Inselspital, University of Bern, Bern, Switzerland

${ }^{2}$ Department of Neurosurgery, Inselspital, University of Bern, Bern, Switzerland

${ }^{3}$ Division of Neuroradiology, Department of Medical Imaging and Division of Neurosurgery, Department of Surgery, University Health Network - Toronto Western Hospital, Toronto, Ontario, Canada

\section{Correspondence to}

Dr Tomas Dobrocky, Department of diagnostic and interventional Neuroradiology, Inselspital Universitatsspital Bern, Bern 3010, Switzerland; tomas. dobrocky@insel.ch

Received 31 August 2020 Revised 23 September 2020 Accepted 26 September 2020 Published Online First 21 October 2020

Check for updates

(c) Author(s) (or their employer(s)) 2021. Re-use permitted under CC BY-NC. No commercial re-use. See rights and permissions. Published by BMJ.

To cite: Dobrocky T,

Piechowiak El, Goldberg J,

et al. J Neurolntervent Surg

2021:13:580-584.

\section{ABSTRACT}

Background Vertebrobasilar dolichoectasia (VBDE) is a rare type of non-saccular intracranial aneurysm, with poor natural history and limited effective treatment options. Visualizing neurovascular microanatomy in patients with VBDE has not been previously reported, but may yield insight into the pathology, and provide important information for treatment planning.

Objective To carry out a retrospective analysis of ultra-high resolution cone-beam computed tomography (UHR-CBCT) in patients with fusiform basilar aneurysms, visualizing neurovascular microanatomy of the posterior circulation with a special focus on the pontine perforators.

Methods UHR-CBCT was performed in seven patients (mean age 59 years; two female) with a VBDE, and in 14 control patients with unrelated conditions.

Results The mean maximum diameter of the fusiform vessel segment was $28 \mathrm{~mm}$ (range 19-36 mm), and the mean length of the segment was $39 \mathrm{~mm}$ (range $15-50 \mathrm{~mm}$ ). In all patients with VBDE, UHR-CBCT demonstrated an absence of perforating arteries in the fusiform arterial segment and a mean of 3.7 perforators arising from the unaffected vessel segment. The network of interconnected superficial circumferential pontine arteries (brainstem vasocorona) were draping around the aneurysm sac. In controls, a mean of 3.6, 2.5, and 1.2 perforators were demonstrated arising from the distal, mid-, and proximal basilar artery, respectively.

Conclusions The absence of pontine perforators in the fusiform vessel segment of VBDE is counterbalanced by recruitment of collateral flow from pontine perforators arising from the unaffected segment of the basilar artery, as well as collaterals arising from the anterior inferior cerebellar artery/posterior inferior cerebellar artery and superior cerebellar artery. These alternative routes supply the superficial brainstem arteries (brainstem vasocorona) and sustain brainstem viability. Our findings might have implications for further treatment planning.

\section{INTRODUCTION}

Vertebrobasilar dolichoectasia (VBDE) is a rare type of intracranial, non-saccular aneurysm, which may become clinically apparent due to compression of the brainstem and cranial nerves, hemorrhagic or ischemic symptoms, and, less frequently, may be diagnosed incidentally. The natural course of the disease is generally poor, with mortality rates of $40 \%$ after 7 years follow-up reported in a large meta-analysis including 440 patients. ${ }^{1}$ In particular, patients presenting with compressive symptoms tend to have an exceedingly poor outcome, with 7/13 (54\%) patients dying due to severe compression, and 8/13 (62\%) experiencing worsening of the mass effect during follow-up. ${ }^{2}$

Despite technical advances in the endovascular approach the treatment of VBDE remains a challenge, with higher complications rates than with saccular aneurysms. Endovascular reconstruction of the fusiform vessel segment with stent-assisted coiling, stent placement alone, flow diverters, and flow reversal with vertebral artery occlusion have been reported in small case series and case reports. ${ }^{3-5}$ Ischemic complications due to thrombosis-namely, perforating arteries supplying the brainstem, remain the major concern irrespective of the technique, but their visualization in vivo remains a challenge and has not been previously reported in VBDE. ${ }^{6}$

Recently, a new generation ultra-high resolution cone-beam computed tomography (UHR-CBCT) has been introduced, enabling visualization of the neurovascular microanatomy in vivo. The main goal of our study was the retrospective analysis of UHR-CBCT in patients with fusiform basilar aneurysms, visualizing neurovascular microanatomy of the posterior circulation, with a special focus on the pontine perforators.

\section{MATERIAL AND METHODS}

Institutional review board approval was obtained for this dual-center study. Informed consent was waived owing to the retrospective nature of the study.

All patients with a VBDE, in whom a UHRCBCT of the posterior circulation between March 2019 and May 2020 was visualized using either the new angiographic system (Artis icono; Siemens, Erlangen, Germany) or between February 2018 and March 2020 using an established angiographic system (AlluraClarity, Philips Healthcare, Best, Netherlands), were included. 
VBDE was defined using the definitions proposed by Flemming et al and had to meet at least one of the following imaging definitions: (1) fusiform aneurysmal dilatation: $1.5 \times$ normal diameter without a definable neck involving a portion of an arterial segment (either vertebral or basilar) with any degree of tortuosity, (2) dolichoectasia: uniform aneurysmal dilatation of the artery $>1.5 \times$ normal involving either the entire basilar or vertebral artery or both with any degree of tortuosity, or (3) transitional: uniform aneurysmal dilatation of the artery $>1.5 \times$ normal involving the vertebral or basilar artery, or both with a superimposed dilatation of a portion of the involved arterial segment. ${ }^{7}$ Acute dissecting aneurysms in two young adults presenting with an extensive subarachnoid hemorrhage and a normal caliber basilar artery were excluded. Patients in whom a UHR-CBCT was performed due to other indications, mainly angiography performed to evaluate patients with nonaneurysmal or CT angiography (CTA)-negative intracranial hemorrhage, served as controls.

\section{Angiography}

Selective intra-arterial digital subtraction angiography was performed under local anesthesia on a biplane, high- resolution angiographic system (Artis icono or AlluraClarity) using Iopamiro 300 (Iopamidol, Bracco, Switzerland) for vessel opacification. The prototype of the Artis icono system was installed in one of the contributing institutions in March 2019 and provides improved and higher spatial resolution using the as40HDR flat-panel detector with a $49 \mathrm{~cm}$ diagonal entrance plane and an active imaging size of $398 \times 293$ and active matrix size of $2586 \times 1904$.

On both systems, digital subtraction angiography images were obtained using a standard program with 2.5 frames per second in the arterial phase, a focal spot size of $0.3 \mathrm{~mm}$, and edge enhancement reconstruction algorithm. A flat-detector zoom format of $32 \times 32 \mathrm{~cm}$ in each plane was used.

After puncture of the common femoral artery a 5 French (Fr) sheath (Terumo, Terumo Medical, Tokyo, Japan) was inserted to secure the access. For angiography of the supra-aortic vessels a 5 Fr diagnostic catheter was advanced over a $0.035^{\prime \prime}$ hydrophilic guide wire (Terumo). In all patients a four-vessel angiogram was obtained to evaluate the intracranial circulation including anteroposterior (AP), lateral, and spin angiograms.

\section{D micro DynaCT technique for Artis icono}

The diagnostic catheter was placed at the origin of the dominant vertebral artery. The catheter was connected to double-head contrast agent injector (Accutron HP-D, Medtron AG, Saarbrücken, Germany) using a designated Y-pressure line with two check valves (Medtron AG) after having purged all air bubbles with sterile saline solution. In intubated patients, the contrast injection was performed under apnea. Awake patients were instructed to hold their breath immediately prior to initiation of the rotational run to reduce artifacts.

During image acquisition a mixture of non-ionic contrast agent $(15 \mathrm{~mL})$ and sterile saline solution $(30 \mathrm{~mL})$ was injected with a flow of $3 \mathrm{~mL} / \mathrm{s}$ (total volume $45 \mathrm{~mL}$, contrast agent concentration $33 \%$, injection time $15 \mathrm{~s}$ ). The amount of contrast agent was adapted to allow adequate vessel opacification during the entire run and prevent any beam hardening artifacts. Continuous fluoroscopic monitoring at a rate of 1 frame/s was used for contrast appearance in the basilar artery (BA) to trigger the rotational run. On appropriate opacification a dedicated UHR-CBCT run with a scan time of $14 \mathrm{~s}$, focusing on the posterior circulation, was performed. During the run the $\mathrm{C}$-arm rotates around the patient over 200 degrees to create a circumferential run of the region of interest. In each run, 500 images with a resolution of $0.14 \mathrm{~mm}$ were obtained. The raw data were then transferred to a dedicated workstation.

\section{D VasoCT for Philips AlluraClarity}

A slightly different protocol was used with the Philips angio. To create a circumferential run the $\mathrm{C}$-arm rotates around the patient over 207 degrees in 20.7 s, acquiring 622 images of the region of interest $(22 \mathrm{~cm}$ field of view). The injector was coupled to the acquisition system, with a fixed $3 \mathrm{~s}$ delay. For good vessel opacification during the run (20.7 scan +3 delay) a mixture of non-ionic contrast agent and sterile saline solution (1:3) was injected with a flow of $3 \mathrm{~mL} / \mathrm{s}$ (total volume $75 \mathrm{~mL}$ ).

\section{Data reconstruction and analysis}

Transversal flat-detector CT reconstructions (arterial mode) were reconstructed at the discretion of the operator. Multiplanar reconstructions with a slice thickness of $0.5 \mathrm{~mm}$ and maximum intensity projections with section thicknesses between 5 and 10 $\mathrm{mm}$ as well as volume rendering reconstructions were sent to the local Picture Archiving and Communicating System (PACS) (R11.4.1, 2009; Philips, Best, Netherlands; Sectra, Linkoping, Sweden; or Coral, Toronto, Canada) and used for perforator analysis.

The clinical information was retrieved from locally maintained databases, and all imaging findings were reviewed by a board-certified neuroradiologist with 9 years of experience (TD) and neuroradiology fellow with 2 years of experience (EBA).

\section{RESULTS}

\section{Vertebrobasilar dolichoectasia group}

In total, UHR-CBCT was available in seven patients with VBDE. Their mean age was 59.0 years $( \pm 6.7)$, and two patients were female. All patients with VBDE demonstrated severe brainstem compression with a mean minimal AP distance of $8 \mathrm{~mm}$ measured on axial images (range $3-12 \mathrm{~mm}$; figures $1 \mathrm{~A}, \mathrm{~B}$ and 2A,B). Surrounding T2 hyperintense brainstem edema was noted on MRI in five patients. The mean maximum diameter of the fusiform vessel segment was $28 \mathrm{~mm}$ (range 19-36 mm), and the mean length of the segment was $39 \mathrm{~mm}$ (range 15-50 mm; table 1). The basilar artery demonstrated an elongated course in all patients.Intramural hematoma was noted in all but one patient (figure 2C), In total, one patient demonstrated a small hyperintense lesion in the brainstem on diffusion weighted imaging, indicating acute ischemia. One patient presented infratentorial superficial siderosis at the 1-year follow-up, indicative of interval hemorrhage.

In all patients with VBDE, UHR-CBCT demonstrated an absence of perforating arteries in the fusiform arterial segment and a mean of 3.7 perforators arising from the unaffected vessel segment. Owing to the mass effect of the aneurysm sac the superficial brainstem arteries were displaced and were draping around the aneurysm.

In one patient the anterior inferior cerebellar artery (AICA) was noted to arise from the fusiform vessel segment, in the remaining patients the AICA territory was supplied by a dominant posterior inferior cerebellar artery (PICA-AICA variant).

\section{Control group}

In total, 14 patients with a mean age of 54.5 years $( \pm 7.9)$ in whom a UHR-CBCT was performed due to unrelated indications were included for reference $(n=11$ CTA-negative intracranial 


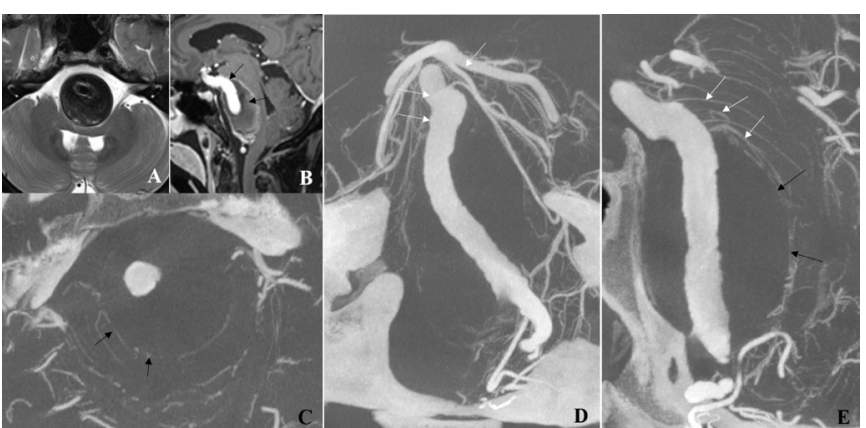

Figure 1 (A) Axial T2-weighted image demonstrating a large vertebrobasilar dolichoectasia with severe mass effect leading to brainstem compression and displacement. (B) Sagittal T1-weighted enhanced image demonstrating the longitudinal extension of the aneurysm and extensive intramural hematoma (black arrows). (C-E) Ultra-high resolution cone-beam computed tomography with maximal intensity reconstructions demonstrating an irregular margin of the fusiform vessel segment in the mid- and lower basilar artery with absence of pontine perforators originating from the fusiform vessel segment. Multiple perforators arising from the basilar artery distal to the aneurysmal segment may be appreciated (white arrows). Note the circumferential pontine arteries (black arrows) which have been displaced due to the mass effect, and seem to be draping around the aneurysm; their patency is maintained by collateral flow from perforators arising from the unaffected segment of the basilar artery, as well as the anterior inferior cerebellar artery/posterior inferior cerebellar artery and superior cerebellar artery.

hemorrhage, $\mathrm{n}=1$ symptomatic basilar artery stenosis; $\mathrm{n}=1$ basilar tip aneurysm, $\mathrm{n}=1$ hemorrhagic dural arteriovenous fistula at the craniocervical junction). In controls, a mean of 3.6, 2.5, and 1.2 perforators were demonstrated arising from the distal, mid, and proximal basilar artery, respectively (figure 3 ). In most of them multiple circumferential pontine arteries coursing along the surface of the pons and small interconnecting branches ('brainstem vasocorona') were appreciated.

\section{DISCUSSION}

The improved spatial resolution available with new-generation angiographic systems incorporating cone-beam CT technology provides valuable insight into the neurovascular microanatomy of the posterior fossa. It demonstrates a complete absence of pontine perforators in the fusiform vessel segment of patients with VBDE. Their absence is counterbalanced by recruitment of collateral flow from pontine perforators arising from the unaffected segment of the BA, as well as collaterals arising from the AICA/PICA and superior cerebellar artery (SCA). These alternative routes supply the superficial brainstem arteries and sustain brainstem viability.

Perforating arteries arising from the BA play a crucial role in the supply of the brainstem, and their compromise may lead to ischemia affecting respiratory and cardiac function, as well as the relay of major motor and sensory functions. Clinicopathologic studies in patients with VBDE have demonstrated circumferential fragmentation of the internal elastic lamina, intimal hyperplasia, and angiogenesis of vasa vasorum, which may result in repetitive intramural hemorrhages and thrombus formation. ${ }^{8}$ According to our results we believe that these destructive vessel wall changes in the fusiform segment progressively lead to obliteration of perforating arteries arising in this vessel segment, and when occurring gradually are balanced by collateral pathways.

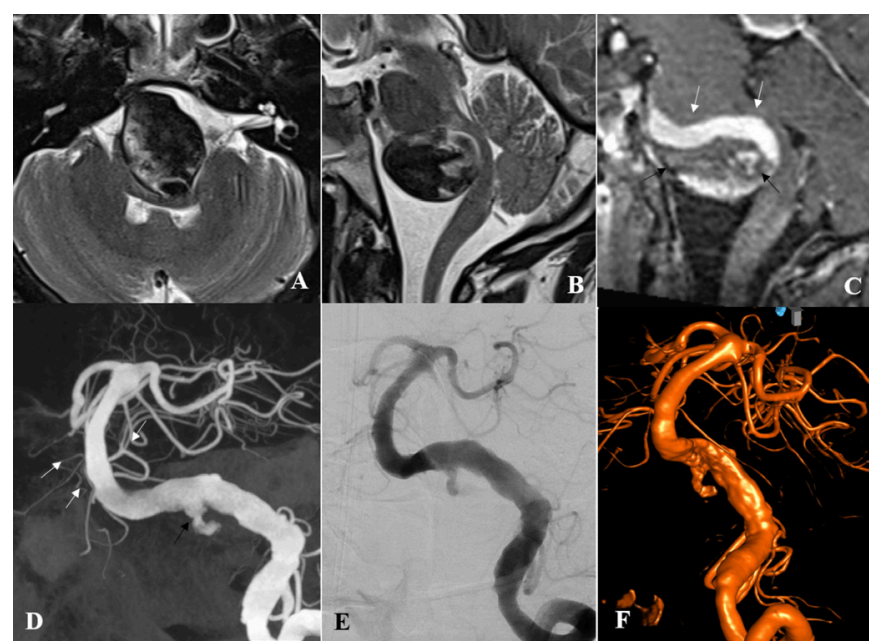

Figure 2 (A, B) Axial and mid-sagittal T2-weighted image demonstrating a large vertebrobasilar dolichoectasia with severe compression of the brainstem and edema. (C) Sagittal T1-weighted enhanced image demonstrating the opacified vessel lumen in the cranial part of the aneurysm (white arrows), and extensive intramural hematoma in the inferior portion (black arrow). (D) Ultra-high resolution cone-beam computed tomography (UHR-CBCT) with coronal maximal intensity reconstructions demonstrating a fusiform aneurysm with incorporation of a $40 \mathrm{~mm}$ segment of the lower and mid-basilar trunk. Note the irregular entry zone of contrast (black arrow) into the thrombosed portion of the aneurysm. There is no evidence of pontine perforators within the fusiform vessel segment. Multiple perforators arising from the distal basilar artery may be appreciated (white arrows). (E) Digital subtraction angiography run in posterior-anterior projection and (F) three-dimensional rotational angiography for comparison show the findings but with significantly less detail and clarity than on UHRCBCT.

In general, pontine perforators are poorly appreciated on standard cross-sectional imaging. including CTA, and conventional 1.5 and 3 Tesla MRA techniques. Recently, compelling results on ultra-high field 7 Tesla MRI have been published, reporting a mean number of $7.14 \pm 2.79$ perforator arteries arising from the basilar artery. ${ }^{9}$ However, ultra-high field MRI is not readily available in clinical routine.

Alternatively, flat-detector CT, first described by Kyriakou et al in $2007,{ }^{10}$ has been used to depict in vivo vascular microanatomy in various intracranial pathologies. ${ }^{11} 12$ The continuing evolution of flat-detector technology has enabled the current rotational angiography systems to provide high acquisition speed coupled with excellent resolution isotropic datasets, and good reconstruction quality. Lescher et al reported that three-dimensional rotation angiography and flat-detector CT reconstructions were superior to $2 \mathrm{D}$ digital subtraction angiography in demonstrating anatomic patterns of circumferential arteries and direct pontine perforators. Furthermore, the authors reported that no zone of basilar artery is free from important side branches, which is in line with the findings in our control population. ${ }^{13}$ On the other hand, according to our results the absence of perforators arising from the fusiform vessel segment seems to be a characteristic feature in patients with VBDE.

Lasjaunias et al regarded the arterial anatomy of the posterior fossa as a transitional pattern between the 'simple' arrangement of the spinal cord and the more 'complex' neocortical pattern. During the embryological development, the BA below the trigeminal point is formed by the fusion of the paired longitudinal 
Table 1 Demographic information on patients with vertebrobasilar dolichoectasia

\begin{tabular}{|c|c|c|c|c|c|c|c|c|c|c|}
\hline & VBDE max. diameter & VBDE length & Intramural hematoma & Brainstem compression & Min. brainstem width & Oedema & Hemorrhage & Siderosis & Ischemia & Ectasia ICA \\
\hline 1 & 36 & 45 & 1 & 1 & 12 & 1 & 1 & 1 & 0 & 1 \\
\hline 2 & 34 & 40 & 1 & 1 & 3 & 1 & 0 & 0 & 0 & 1 \\
\hline 3 & 30 & 50 & 1 & 1 & 5 & 1 & 0 & 0 & 0 & 0 \\
\hline 4 & 33 & 38 & 1 & 1 & 10 & 1 & 0 & 0 & 0 & 0 \\
\hline 5 & 21 & 43 & 1 & 1 & 15 & 1 & 0 & 0 & 1 & 1 \\
\hline 6 & 19 & 15 & 0 & 1 & 11 & 0 & 0 & 0 & 0 & 0 \\
\hline 7 & 26 & 45 & 1 & 1 & 6 & 0 & 0 & 0 & 0 & 0 \\
\hline
\end{tabular}

All measurements are provided in millimeters $(\mathrm{mm}) .1=\mathrm{Yes}, 0=\mathrm{No}$

ICA, internal carotid artery; VBDE, vertebrobasilar dolichoectasia.

neural arteries-similar to the formation of the anterior spinal artery, whereas the BA cranial to the trigeminal point is formed by the fusion of the paired caudal divisions of the embryologic internal carotid artery (ICA). Thus, the junction between the two systems (simplified spinal system and complex cortical system) is near the trigeminal remnant site on the BA. Being thus a transitional artery between the two systems, there is a certain degree of resemblance to the ASA system, and the pontine perforators may be regarded as counterparts of the coronary and sulcocommissural arteries. ${ }^{14}$ The BA, vertebrobasilar confluence, and the PICA give rise to a considerable number of perforators supplying the brainstem and the pontomedullary junction. Conversely, there is a paucity of perforators arising from the distal vertebral artery between its entry through the dura mater and the PICA origin. Distal vertebral perforators are reported in only $4 \%$ of individuals in an anatomical study by Mercier et al. ${ }^{15}$

Detailed anatomical studies have demonstrated a considerable amount of artery-to-artery anastomoses between the superficial brainstem arteries ('brainstem vosocorona'), ${ }^{16}$ and may be considered the equivalent of the rich arterial network coursing on the surface of the spinal cord known as the arterial vasocorona.

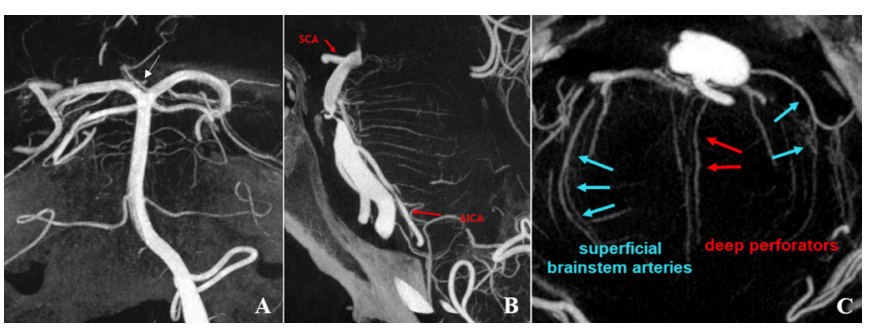

Figure 3 (A) A patient presenting with thunderclap headache and perimesencephalic subarachnoid hemorrhage on CT (not shown). Ultra-high resolution cone-beam computed tomography (UHR-CBCT) acquired during injection of the left vertebral artery, Maximum intensity projection images in the coronal plane demonstrating multiple perforating arteries originating from the mid- and distal basilar artery. Note: artery of Percheron (arrow) originating as a solitary arterial trunk from the P1 segment on the left supplying the paramedian thalami and parts of the rostral midbrain bilaterally. (B) UHR-CBCT in the sagittal projection in a patient presenting with acute pontine ischemia (not shown) due to an underlying high-grade mid-basilar stenosis. Multiple deep perforators and several circumferential pontine arteries coursing along the surface of the pons and small interconnecting branches ('brainstem vasocorona') may be appreciated. (C) UHR-CBCT of the same patient in the axial plane demonstrating the course of circumferential pontine arteries running along the surface of the pons and deep perforating branches running in the midline. AICA, anterior inferior cerebellar artery; SCA, superior cerebellar artery.
As reported by Lindsberg in patients with acute basilar artery occlusion, the PICA is a strong collateral to the AICA and SCA and thus to the brainstem perforating arterioles which may maintain brainstem vitality for significant periods if the clot does not gradually extend to block the perforating arteries. ${ }^{17}$ Likewise in patients with VBDE the gradual obliteration of perforating arteries at the level of the fusiform vessel segment is probably compensated by a considerable network of anastomoses ('brainstem vosocorona'), recruiting collaterals arising in the unaffected vessel segments of the basilar artery as well as AICA/PICA and SCA (figure 4). In patients in whom the AICA is incorporated within the fusiform segment, based on our data the thrombotic risks after flow diverter deployment remain unclear.

In a large meta-analysis looking at the natural history of VBDE, including 827 patients with 5093 patient-years, the authors reported an overall annual mortality rate of 13\%/year (95\% CI $8 \%$ to 19\%). Patients with fusiform aneurysms had a higher mortality rate than those with dolichoectatic aneurysmal dilatation $(12 \mathrm{vs} 8 \%, \mathrm{p}=0.11)$. The overall growth rate was $6 \%$ / year (95\% CI $4 \%$ to $13 \%)$, and the overall rupture rate was $3 \% /$ year (95\% CI $1 \%$ to $5 \%$ ). ${ }^{18}$ As stated previously, patients presenting with compressive symptoms, in particular, tend to show exceedingly poor outcome. Chen et al reported that a BA diameter $\geq 5.3 \mathrm{~mm}$ was independently associated with increased
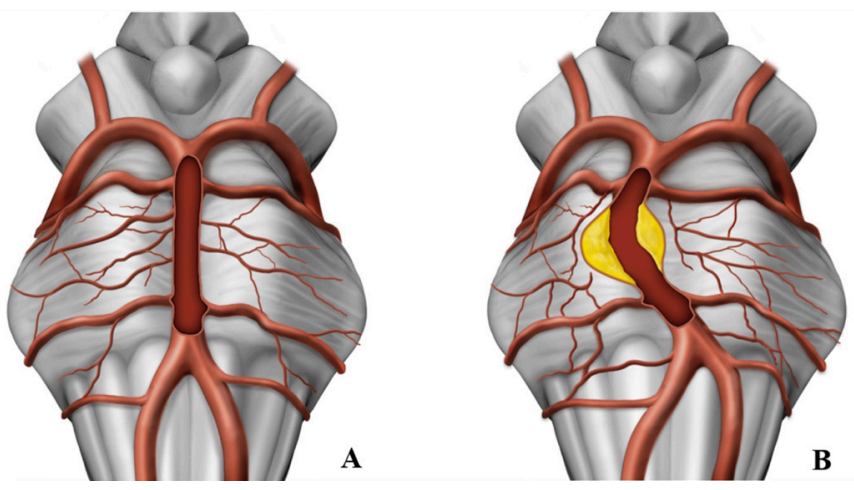

Figure 4 (A) Illustration of a normal basilar artery with several circumferential pontine arteries coursing along the surface of the pons and small interconnecting branches ('brainstem vasocorona'; arrowheads). (B) A large fusiform basilar artery aneurysm with circumferential intramural hematoma. Note the absence of pontine perforators within the fusiform vessel segment. Obliteration of perforating arteries at the level of the fusiform vessel segment is compensated by the brainstem vosocorona, recruiting collaterals arising in the unaffected vessel segments of the basilar artery as well as from the anterior internal cerebral artery/posterio internal cerebral artery and superior cerebellar artery. 
stroke recurrence in patients with VBDE on multivariate analysis $(\mathrm{HR}=4.744 ; 95 \% \mathrm{CI}, 1.718$ to $13.097 ; \mathrm{p}=0.003) .{ }^{19}$ This is mainly thought to relate to compromise of basilar perforating arteries, either due to thrombosis or due to their compression or stretching.

Initially the anatomic aspects and the large number of perforators were believed to preclude neurointerventional treatment of VBDE. However, promising results of endovascular reconstruction of the fusiform segment with flow diverters (FDs) and stentassisted coiling have been reported in multiple case reports and case series. ${ }^{20-24}$ Fiorella et al reported treatment of a giant midbasilar trunk aneurysm with seven serially placed, telescoping FDs with complete occlusion. ${ }^{25}$ Nevertheless, in a recent metaanalysis the overall complication rate was higher for FDs than with stent-assisted coiling (18\% vs 6\%), including higher chance for stroke $(13 \%$ vs $5 \%, p=0.04) .{ }^{26}$ The safety and efficacy of endovascular treatment of patients with VBDE is dependent on the size of the lesion, the patient's overall clinical condition, and the treatment modality used.

Further development of UHB-СВCT has enabled visualization of the neurovascular microanatomy in vivo unprecedented by previous techniques. It thus leads to a better understanding of the pathology and as such has important implications on therapy. Its implementation into clinical routine in selected pathologies may be helpful. Nevertheless, VBDE continues to be a challenging pathology for endovascular therapy, but based on our results, the risk of compromising perforators in the fusiform segment itself seems to be low. Attention has to be paid to the perforators arising from the adjacent normal proximal and distal basilar artery segments.

The major strength of our study is a meticulous description of a protocol for improved in vivo visualization of microanatomy using UHB-CBCT and presentation of illustrative case examples of patients with a VBDE. The limitation of this study is the small number of patients, its retrospective nature, and its multicenter design.

\section{CONCLUSION}

In patients with VBDE the fusiform vessel segment is characterized by the absence of pontine perforators, which is in contrast to controls, in whom no zone of the basilar artery is free of perforators. In patients with VBDE, perforating arteries arising in the unaffected segment of the basilar artery, as well as AICA/ PICA, and SCA, supply the network of superficial brainstem arteries which maintain brainstem viability. UHR-СВCT leads to a better understanding of the pathology, and its implementation into clinical routine has important implications and may help to guide further therapies.

Acknowledgements We thank Anja Giger, Department of Neurosurgery, University of Bern, Inselspital, for the provided illustrations. We thank the Neuroangio teams from Bern and Toronto, in particular our radiology technicians Marco Matzinger, Thierry Horisberger, and Nicole Cancelliere.

Contributors TD, VMP, PM designed the study concept. TD, EIIP, EBA, PM were responsible for data acquisition. TD, EBA performed image analysis. The lead author (TD) wrote the first draft of the manuscript. All authors were involved in data interpretation and revising the manuscript for intellectual content.

Funding The authors have not declared a specific grant for this research from any funding agency in the public, commercial or not-for-profit sectors.

Competing interests None declared.

Patient consent for publication Not required.

Ethics approval Institutional review board approval was obtained for this dualcenter study. Informed consent was waived owing to the retrospective nature of the study.
Provenance and peer review Not commissioned; externally peer reviewed.

Data availability statement Data are available upon reasonable request. Raw data of all patients included in this study can be made available upon request to the corresponding author and after clearance by the local ethics committee.

Open access This is an open access article distributed in accordance with the Creative Commons Attribution Non Commercial (CC BY-NC 4.0) license, which permits others to distribute, remix, adapt, build upon this work non-commercially, and license their derivative works on different terms, provided the original work is properly cited, appropriate credit is given, any changes made indicated, and the use is non-commercial. See: http://creativecommons.org/licenses/by-nc/4.0/.

\section{ORCID iDs}

Tomas Dobrocky http://orcid.org/0000-0002-6167-3343

Eike I Piechowiak http://orcid.org/0000-0001-5609-0998

Johannes Kaesmacher http://orcid.org/0000-0002-9177-2289

\section{REFERENCES}

1 Shapiro M, Becske T, Riina HA, et al. Non-saccular vertebrobasilar aneurysms and dolichoectasia: a systematic literature review. J Neurointerv Surg 2014;6:389-93.

2 Wang J, Jia L, Yang $X$, et al. Outcomes in symptomatic patients with vertebrobasilar dolichoectasia following endovascular treatment. Front Neurol 2019;10:610.

3 Wu X, Xu Y, Hong B, et al. Endovascular reconstruction for treatment of vertebrobasilar dolichoectasia: long-term outcomes. AJNR Am J Neuroradiol 2013;34:583-8.

4 Tan LA, Moftakhar R, Lopes DK. Treatment of a ruptured vertebrobasilar fusiform aneurysm using pipeline embolization device. J Cerebrovasc Endovasc Neurosurg 2013;15:30.

5 Bhogal P, Pérez MA, Ganslandt O, et al. Treatment of posterior circulation nonsaccular aneurysms with flow diverters: a single-center experience and review of 56 patients. J Neurointerv Surg 2017;9:471-81.

6 Klisch J, Turk A, Turner R, et al. Very late thrombosis of flow-diverting constructs after the treatment of large fusiform posterior circulation aneurysms. AJNR Am J Neuroradiol 2011:32:627-32.

7 Flemming KD, Wiebers DO, Brown RD, et al. Prospective risk of hemorrhage in patients with vertebrobasilar nonsaccular intracranial aneurysm. J Neurosurg 2004; 101:82-7.

8 Nakatomi H, Segawa H, Kurata A, et al. Clinicopathological study of intracranial fusiform and dolichoectatic aneurysms : insight on the mechanism of growth. Stroke 2000:31:896-900.

9 Kang C-K, Park C-A, Kim K-N, et al. Non-Invasive visualization of basilar artery perforators with 7T Mr angiography. J Magn Reson Imaging 2010;32:544-50.

10 Kyriakou Y, Struffert T, Dörfler A, et al. Grundlagen Der Flachdetektor-CT (FD-CT). Radiologe 2009;49:811-9.

11 Amano T, Sato M, Matsumaru Y, et al. Intra-arterial contrasted cone-beam computed tomography assessment of vessels distal from occluded site in acute ischemic stroke with major vessel occlusion. Neurol Med Chir 2017;57:292-8.

12 Safain MG, Rahal JP, Patel S, et al. Superior performance of cone-beam CT angiography in characterization of intracranial atherosclerosis. J Neurosurg 2014;121:441-9.

13 Lescher S, Samaan T, Berkefeld J. Evaluation of the pontine perforators of the basilar artery using digital subtraction angiography in high resolution and $3 \mathrm{D}$ rotation technique. AJNR Am J Neuroradiol 2014;35:1942-7.

14 Lasjaunias P, Berenstein A, ter Brugge KG. Clinical vascular anatomy and variations. 2nd ed. Springer, 2001.

15 Mercier PH, Brassier G, Fournier HD, et al. Vascular microanatomy of the pontomedullary junction, posterior inferior cerebellar arteries, and the lateral spinal arteries. Interv Neuroradiol 2008;14:49-58.

16 Duvernoy HM. Human brain stem vessels including the pineal gland and information on brain stem infarction. 2nd ed. Springer Berlin: Berlin, 1999.

17 Lindsberg PJ, Pekkola J, Strbian D, et al. Time window for recanalization in basilar artery occlusion: speculative synthesis. Neurology 2015;85:1806-15.

18 Nasr DM, Flemming KD, Lanzino G, et al. Natural history of vertebrobasilar dolichoectatic and fusiform aneurysms: a systematic review and meta-analysis. Cerebrovasc Dis 2018;45:68-77.

19 Chen Z, Zhang S, Dai Z, et al. Recurrent risk of ischemic stroke due to vertebrobasilar dolichoectasia. BMC Neurol 2019;19:4-11.

20 Natarajan SK, Lin N, Sonig A, et al. The safety of pipeline flow diversion in fusiform vertebrobasilar aneurysms: a consecutive case series with longer-term follow-up from a single us center. J Neurosurg 2016;125:111-9.

21 Siddiqui $\mathrm{AH}$, Abla $\mathrm{AA}$, Kan $\mathrm{P}$, et al. Panacea or problem: flow diverters in the treatment of symptomatic large or giant fusiform vertebrobasilar aneurysms. J Neurosurg 2012;116:1258-66.

22 Jia L, Wang J, Zhang L, et al. Pediatric patient with a giant vertebrobasilar dissecting aneurysm successfully treated with three pipeline embolization devices. Front Neurol 2020;11:1-4. 
23 Pumar JM, Garcia-Dorrego R, Nieto A, et al. Vascular reconstruction of a fusiform basilar aneurysm with the silk embolization system. J Neurointerv Surg 2010;2:242-4.

24 He X, Duan C, Zhang J, et al. The safety and efficacy of using large woven stents to treat vertebrobasilar dolichoectasia. J Neurointerv Surg 2019;11:1162-6.
25 Fiorella $\mathrm{D}$, Kelly $\mathrm{ME}$, Albuquerque $\mathrm{FC}$, et al. Curative reconstruction of a giant midbasilar trunk aneurysm with the pipeline embolization device. Neurosurgery 2009;64:212-7.

26 Domingo RA, Tripathi S, Perez-Vega C, et al. Treatment of posterior circulation non-saccular aneurysms with flow diversion versus stent-assisted coiling: a systematic review and meta-analysis. J Neurointerv Surg 2020. doi:10.1136/ neurintsurg-2020-016294. [Epub ahead of print: 10 Jul 2020]. 